\title{
The role of radar in predicting and preventing asteroid and comet collisions with Earth
}

\author{
Steven J. Ostro \\ Jet Propulsion Laboratory, California Institute of Technology \\ Jon D. Giorgini \\ Jet Propulsion Laboratory, California Institute of Technology
}

\section{Introduction}

The current Spaceguard Survey classifies each known near-Earth asteroid (NEA) as either non-threatening or deserving of additional astrometric attention. For any possibly threatening object, the dominant issues are the uncertainty in its trajectory and physical nature as well as what can be done to reduce that uncertainty. Morrison et al. (2002) note that

From the standpoint of an allocator of society's resources, an uncertain threat calls for adaptive policies, delaying potentially costly action but informing later decision by investing in uncertainty-reduction measures. In the context of the NEO impact hazard, this means avoiding the costs of standing organizational structures and capital expenditures until a threat materializes....

Thus reduction in uncertainty is tantamount to ensuring that unnecessary costs are avoided and that necessary actions are undertaken with adequate warning.

Ground-based radar is a knowledge-gathering tool that is uniquely able to shrink uncertainty in NEO trajectories and physical properties. The power of radar stems largely from the precision of its measurements (Table 3.1). The resolution of echoes in time delay (range) and Doppler frequency (radial velocity) is often of order $1 / 100$ the extent of a kilometer-sized target, so several thousand radar image pixels can be placed on the target. Delay-Doppler positional measurements often have a fractional precision finer than $1 / 10000000$, comparable to sub-milliarcsecond optical astrometry.

The single-date signal-to-noise ratio (SNR) of echoes, a measure of the number of useful imaging pixels placed on a target by a given radar data set, depends primarily on the object's distance and size. Figure 3.1 shows nominal values of

Mitigation of Hazardous Comets and Asteroids, ed. M. J. S. Belton, T. H. Morgan, N. H. Samarasinha, and D. K. Yeomans. Published by Cambridge University Press. (c) Cambridge University Press 2004. 
Table 3.1 Fractional precision of NEA radar measurements ${ }^{a}$

\begin{tabular}{lll}
\hline \hline & Range $(\mathrm{m})$ & Radial velocity $\left(\mathrm{m} \mathrm{s}^{-1}\right)$ \\
\hline Best radar resolution & $\sim 10$ & $\sim 0.0001$ \\
Echo dispersion & $\sim 1000$ & 0.01 to 10 \\
Astrometric "location" & $\sim 10000000$ & $\sim 10000$ \\
\hline \hline
\end{tabular}

$a$ The optimal resolution of radar measurements of the distribution of echo power in time delay (range) and Doppler frequency (radial velocity) for observations of a large NEA is compared with the scale of the object's delay-Doppler extent and location.

SNR for Arecibo and Goldstone. Notwithstanding the heroic efforts by Zaitsev and colleagues in Russia and several intercontinental asteroid radar demonstrations involving Goldstone or Arecibo transmissions with reception of asteroid echoes in Japan, Spain, and Italy, the world's only effective NEO radars are at Arecibo and Goldstone, whose declination windows are $-1^{\circ}$ to $38^{\circ}$ and $>-40^{\circ}$, respectively. However, given the historical funding difficulties experienced by those two systems (Beatty 2002), the future of radar astronomy cannot be taken for granted. Time will tell whether the USA will opt to maintain, much less improve, the current Arecibo and Goldstone radar telescopes.

In this chapter, we examine how our current radar capabilities might help at each stage of detecting and mitigating an impact hazard encountered during this century. See Ostro (1994) for a discussion of radar's role in hazard mitigation written a decade ago, Ostro et al. (2002) for a review of asteroid radar astronomy, and Harmon et al. (1999) for a review of comet radar astronomy.

\section{Post-discovery astrometric follow-up}

Once an asteroid is discovered, its orbital motion must be followed well enough to permit reliable prediction and recovery at the next favorable apparition. As of April 2004, 41\% of the 595 identified Potentially Hazardous Asteroids (PHAs) are lost in the sense that the three-standard-deviation uncertainty in the time of the next close-approach exceeds \pm 10 days, corresponding roughly to a plane-of-sky angular uncertainty greater than $90^{\circ}$. (A PHA is defined by NASA's Jet Propulsion Laboratory as an object having a minimum orbit intersection distance with the Earth $\leq 0.05 \mathrm{AU}$ and an absolute visual magnitude $\mathrm{H} \leq 22$.)

The first asteroid radar astrometry was obtained in 1968 (for 1566 Icarus) (Goldstein 1968; Pettengill et al. 1969). Prior to those observations, from simulations designed to evaluate the usefulness of optical and radar astrometry of Icarus in disclosing relativistic effects, Shapiro et al. (1968) concluded that radar data would 

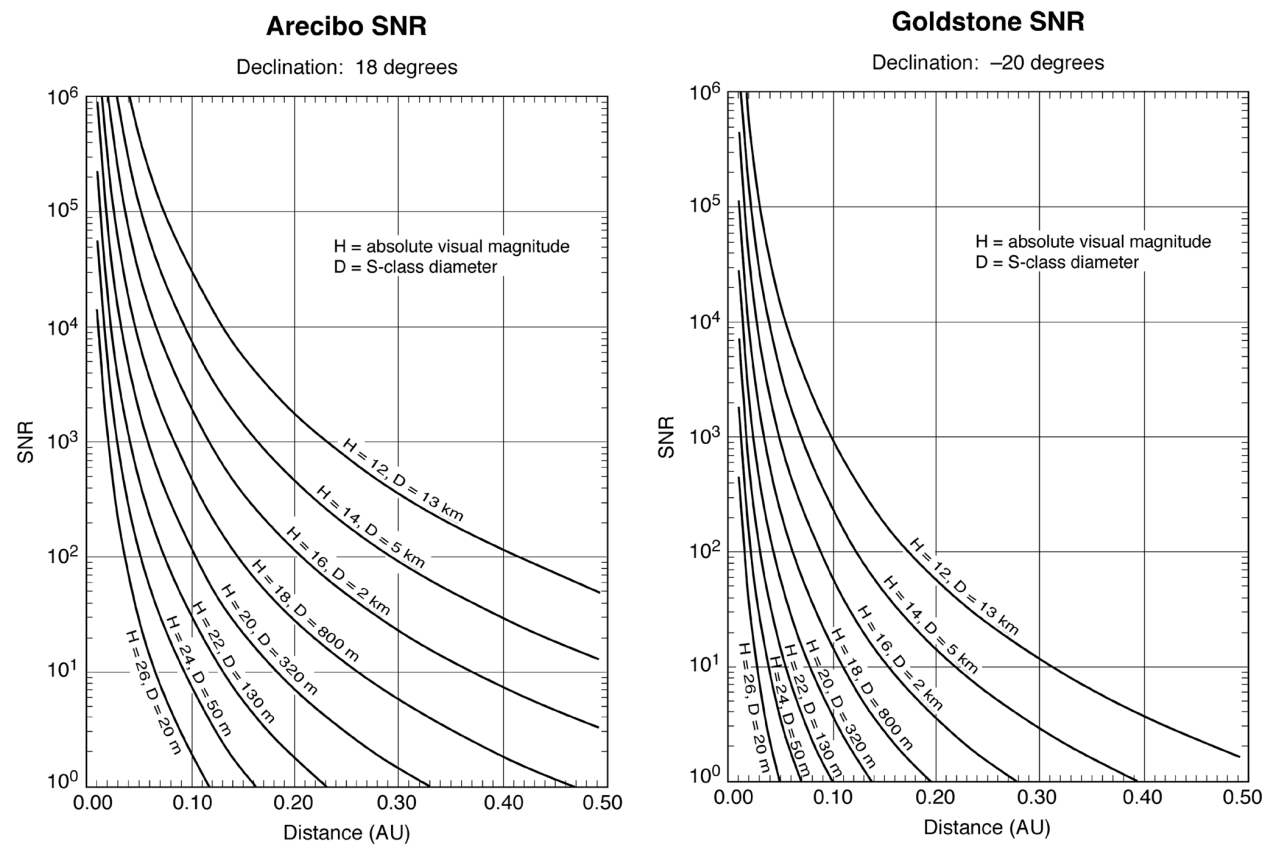

Figure 3.1 Predictions of the single-date signal-to-noise ratio (SNR): (a) for Arecibo echoes from asteroids at declination $18^{\circ}$, and (b) for Goldstone echoes from asteroids at declination $-20^{\circ}$, as a function of the target's distance and absolute visual magnitude (converted to diameter by assuming an S-class optical albedo of 0.16) (Zellner 1979). Other assumptions include a 10\% radar albedo, an equatorial view, a 4-h rotation period, and optimal values for system parameters. Plots for other declinations and distances are on the Internet (Ostro 2004a).

make an impressive contribution to the improvement of estimates of that asteroid's orbit. Two decades later, the potential of delay-Doppler measurements for smallbody orbit refinement was examined comprehensively in a series of Monte Carlo simulations carried out by Yeomans et al. (1987). They showed that a single radar detection of a newly discovered NEA shrinks the instantaneous positional uncertainty at the object's next close approach by orders of magnitude with respect to an optical-only orbit, thereby preventing "loss" of the object. These conclusions have been substantiated quantitatively in the years since through comparison of radar+optical with optical-only positional predictions for recoveries of NEAs during the past decade (Table 3.2): The radar-based predictions historically have had pointing errors that average 310 times smaller than their optical-only counterparts, dramatically facilitating recovery.

Furthermore, radar astrometry can significantly reduce ephemeris uncertainties even for an object whose optical astrometry spans many decades. For example, radar measurements of 1862 Apollo (Ostro et al. 1991) at Arecibo showed that the object's optical-astrometry-based orbit, although based on 49 years of data, had 
Table 3.2 Residuals for past NEA recoveries ${ }^{a}$

\begin{tabular}{llrrr}
\hline \hline Object & Recovery date & $\mathrm{O}$ & $\mathrm{R}$ & $\mathrm{O} / \mathrm{R}$ \\
\hline 1989 PB (4769 Castalia) & May 1990 & $24^{\prime \prime}$ & $0.4^{\prime \prime}$ & 60 \\
1991 AQ & Sep 1994 & $57^{\prime \prime}$ & $0.1^{\prime \prime}$ & 380 \\
1986 DA (6178) & Oct 1994 & $56^{\prime \prime}$ & $0.9^{\prime \prime}$ & 60 \\
1991 JX (6489 Golevka) & Mar 1995 & $3600^{\prime \prime}$ & $4.6^{\prime \prime}$ & 780 \\
1989 JA (7335) & Oct 1996 & $196^{\prime \prime}$ & $99.3^{\prime \prime}$ & 2 \\
1986 JK (14827 Hypnos) & Apr 2000 & $114^{\prime \prime}$ & $0.1^{\prime \prime}$ & 910 \\
1998 ML14 & Nov 2002 & $125^{\prime \prime}$ & $0.5^{\prime \prime}$ & 250 \\
1990 OS & Jun 2003 & $50477^{\prime \prime}$ & $3200^{\prime \prime}$ & 16 \\
\hline \hline
\end{tabular}

${ }^{a}$ Here $\mathrm{O}$ represents the positional offset (the observed position at recovery minus the predicted position) for a pre-recovery orbit solution incorporating only optical astrometry. $\mathrm{R}$ represents the residual for a pre-recovery orbit solution using radar combined with optical. $\mathrm{O} / \mathrm{R}$ is the ratio of residuals for the two cases and is a measure of the relative reduction in position error when radar astrometry is included in the orbit solution.

a range error of $3750 \pm 2 \mathrm{~km}$. (See also the discussion of 1950 DA below.) The reduced uncertainties of a radar orbit can also aid recovery at fainter magnitudes. For example, 1998 ML14 was recovered (Table 3.2) at magnitude 21.2, only 0.5 arcseconds from the position predicted from a radar+optical orbit. The optical-only orbit would have suggested a search for the faint object 125 arcseconds away from the actual position.

For NEAs observed only during their discovery apparition, one can predict the uncertainty in the location during the next opportunity for optical observation, and hence the area of the sky for a search having a given likelihood of success. Table 3.3 lists the total sky area for the three-standard-deviation orbit-determination uncertainties mapped onto the sky at the next favorable Earth-based recovery date (which we define as the next time when the apparent visual magnitude is less than 20 during reasonable sky-brightness conditions) for both an optical-only orbit and a radar+optical orbit, for seven NEAs. Radar shrinks the required sky search area by an average factor of 2642, dramatically facilitating recovery. For six other objects in the table, the optical-only and radar+optical orbits are so different that the nominal recovery dates are months or years apart. Since the radar+optical solutions would be expected to be the more accurate, reliance on an optical-only solution would be unlikely to lead to recovery.

\section{Window of predictability}

A goal of the Spaceguard Survey is to provide as much warning as possible of any possibly dangerous approach of NEAs as large as 1 kilometer. However, since 


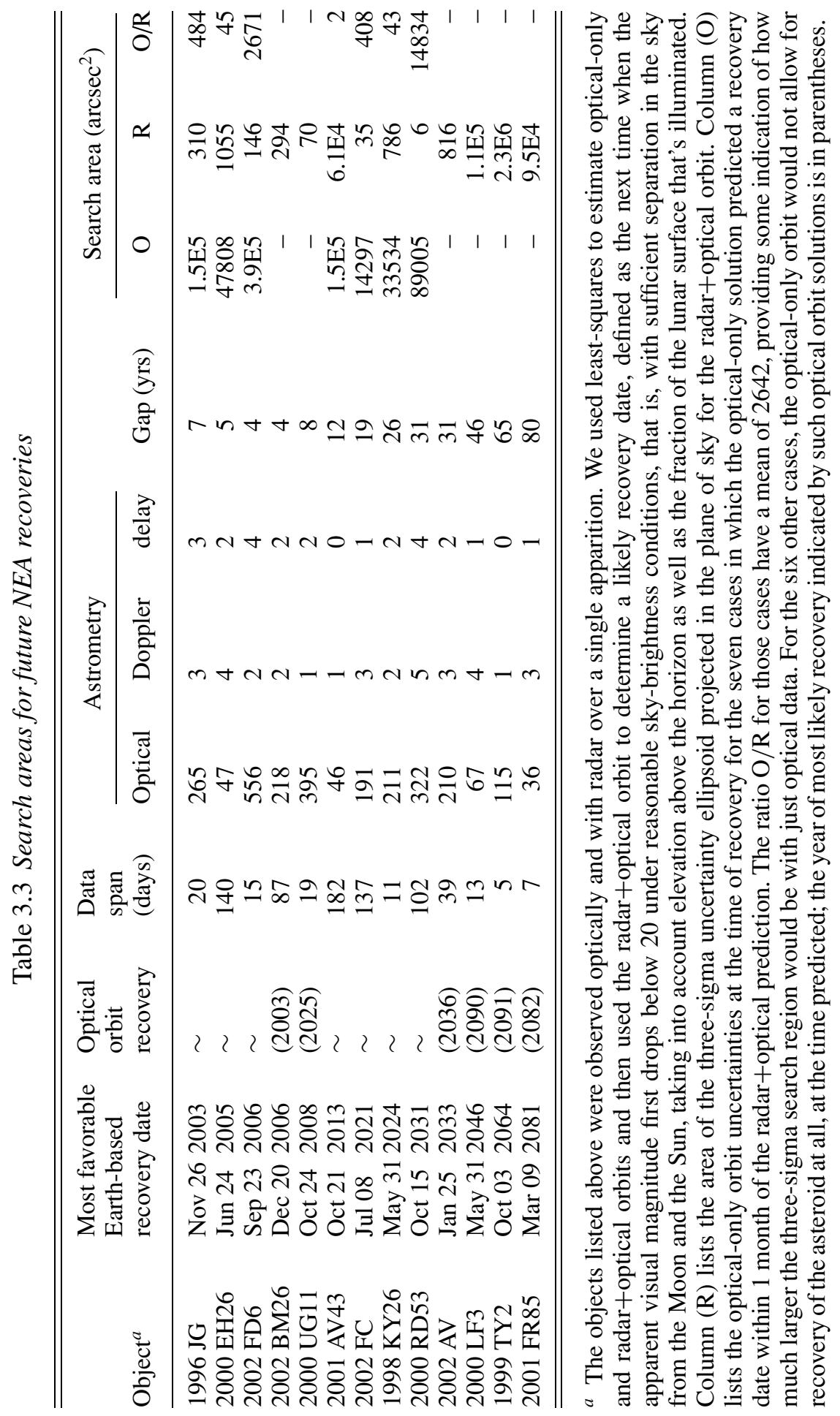


an orbit estimate is based on a least-squares fit to measurements of an asteroid's position over a small portion of its orbit, knowledge of the future trajectory generally is limited by statistical uncertainties that increase with the length of time from the interval spanned by astrometric measurements. Trajectory uncertainties are greatest and grow most rapidly during close planetary encounters, as the steeper gravity field gradient differentially affects the volume of space centered on the nominal orbit solution within which the asteroid is statistically located. Eventually the uncertainty region grows so large, typically within the orbit plane and along the direction of motion, that the prediction becomes meaningless.

Current ground-based optical astrometric measurements typically have angular uncertainties of between 0.2 and 1.0 arcsec (a standard deviation of 0.5 to 0.8 arcsec is common), corresponding to tens or hundreds or thousands of kilometers of uncertainty for any given measurement, depending on the asteroid's distance. Radar can provide astrometry referenced to the asteroid's center of mass, with uncertainties as small as $\sim 10 \mathrm{~m}$ in range and $\sim 1 \mathrm{~mm} \mathrm{~s}^{-1}$ in range rate. Since those measurements are orthogonal to plane-of-sky angular measurements and have relatively fine fractional precision, they offer substantial leverage on an orbit solution and normally extend NEO trajectory predictability intervals far beyond what is possible with optical data alone.

Let us define the window of predictability as the interval over which an object's Earth close approaches can be reliably known at the three-sigma level of confidence. Table 3.4 lists optical-only and radar+optical predictability windows for all radardetected PHAs. For objects observed only during their discovery apparition, radar has enlarged the total window of predictability (past and future) by an average factor of eight, from 145 years for solutions based only on optical data to 1196 years when radar was included in the orbit solution. On average, radar has added 367 more years to the window of accurate future predictions.

When radar astrometry is excluded from the 29 single-apparition PHA radar+optical orbit solutions, $41 \%$ cannot have their next close approach predicted within the adopted confidence level using only the single apparition of optical data. This is the same percentage seen in the total population of PHAs. Radar astrometry obtained in these cases adds an average of 500 years of statistical confidence to their Earth encounter predictions, preventing them from being lost. For 2000 RD53 and 1999 FN53, the extension is through the end of this millennium.

We see that a discovery-apparition orbit solution containing radar astrometry can be compared favorably to a multiple-apparition, optical-only solution. As an example, 1998 ML14 is listed in both single and multiple apparition categories to show the effect of including the first six optical observations from the November 2002 recovery, which lengthened the data arc from 7 months to 5 years and lengthened the optical-only total knowledge window from 364 to 1721 years. By comparison, 


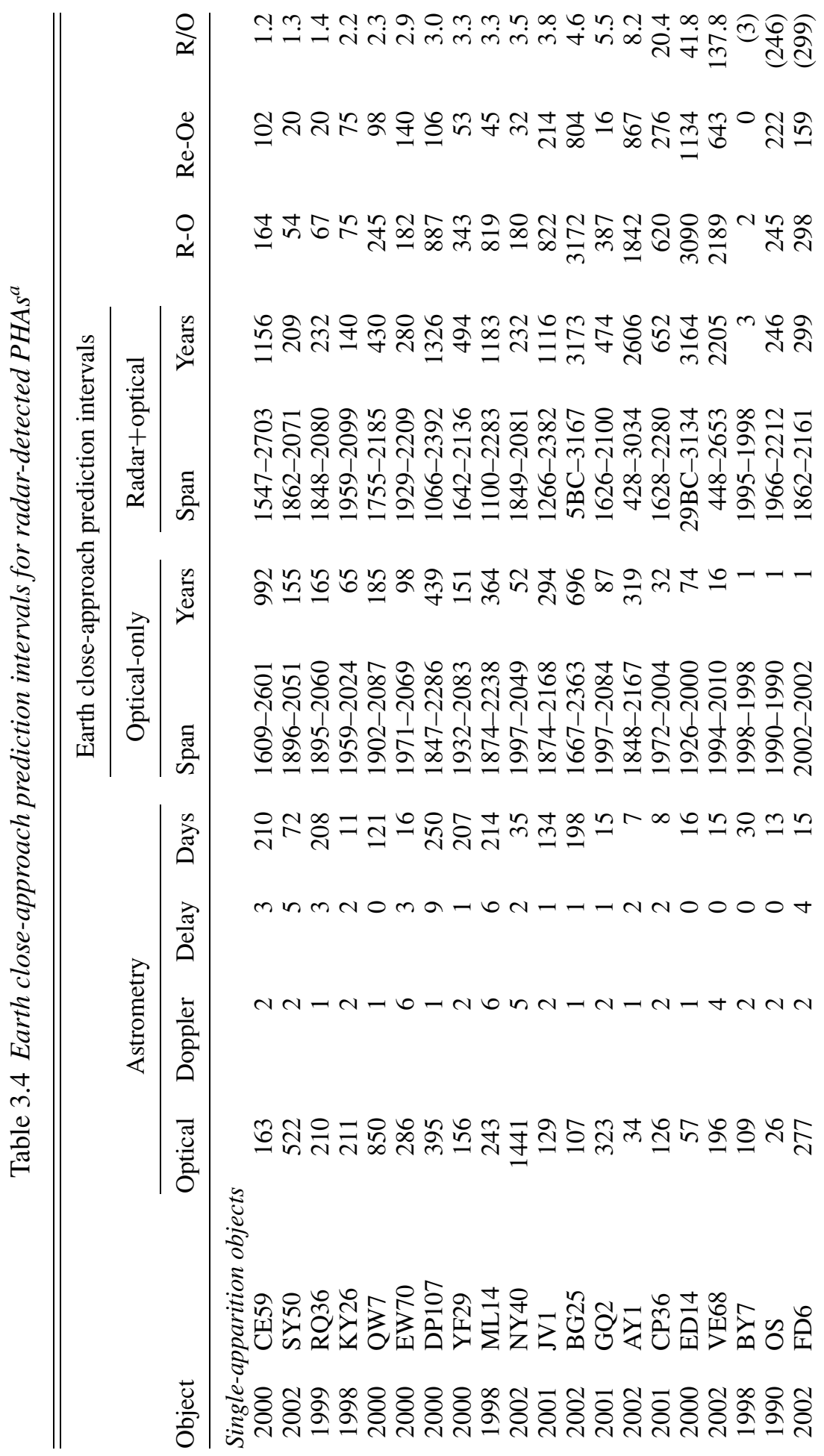




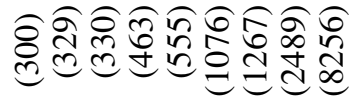

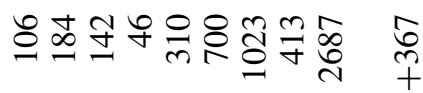

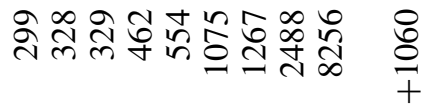

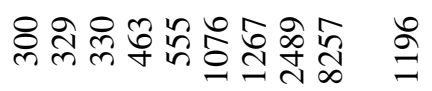

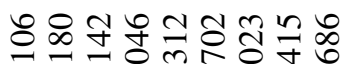

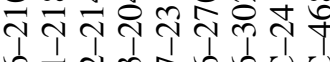

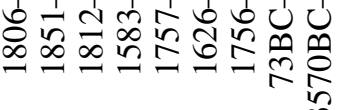

ーーーーーーーー

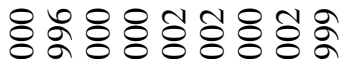
क्रे क्षंत्र

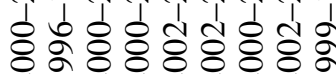

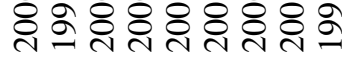

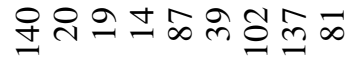

Nm $n$ N

$\forall m-60 m$ n

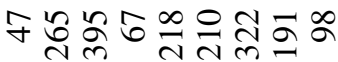

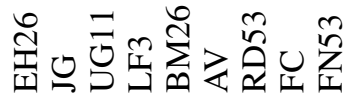

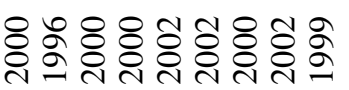

잉

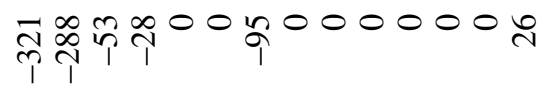

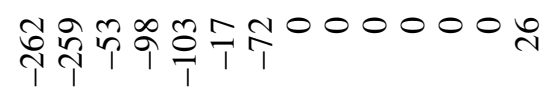

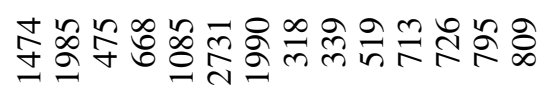

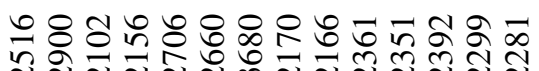

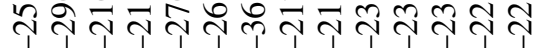

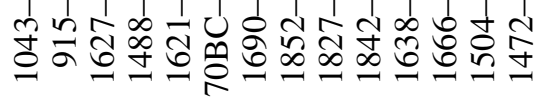

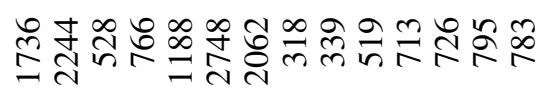
ஸ் ๙

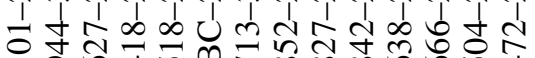

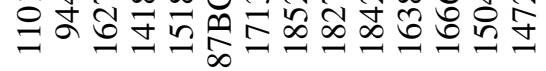

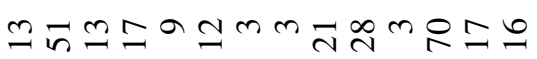

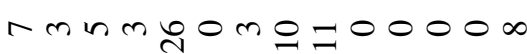

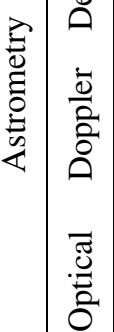
$n+00$ drปิ 芯

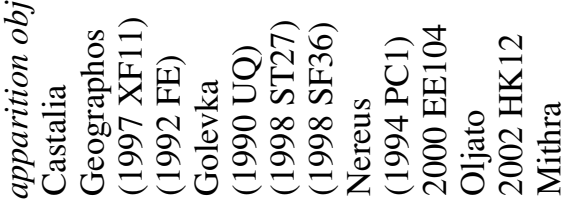

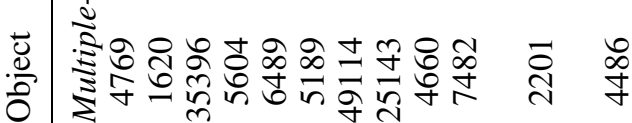




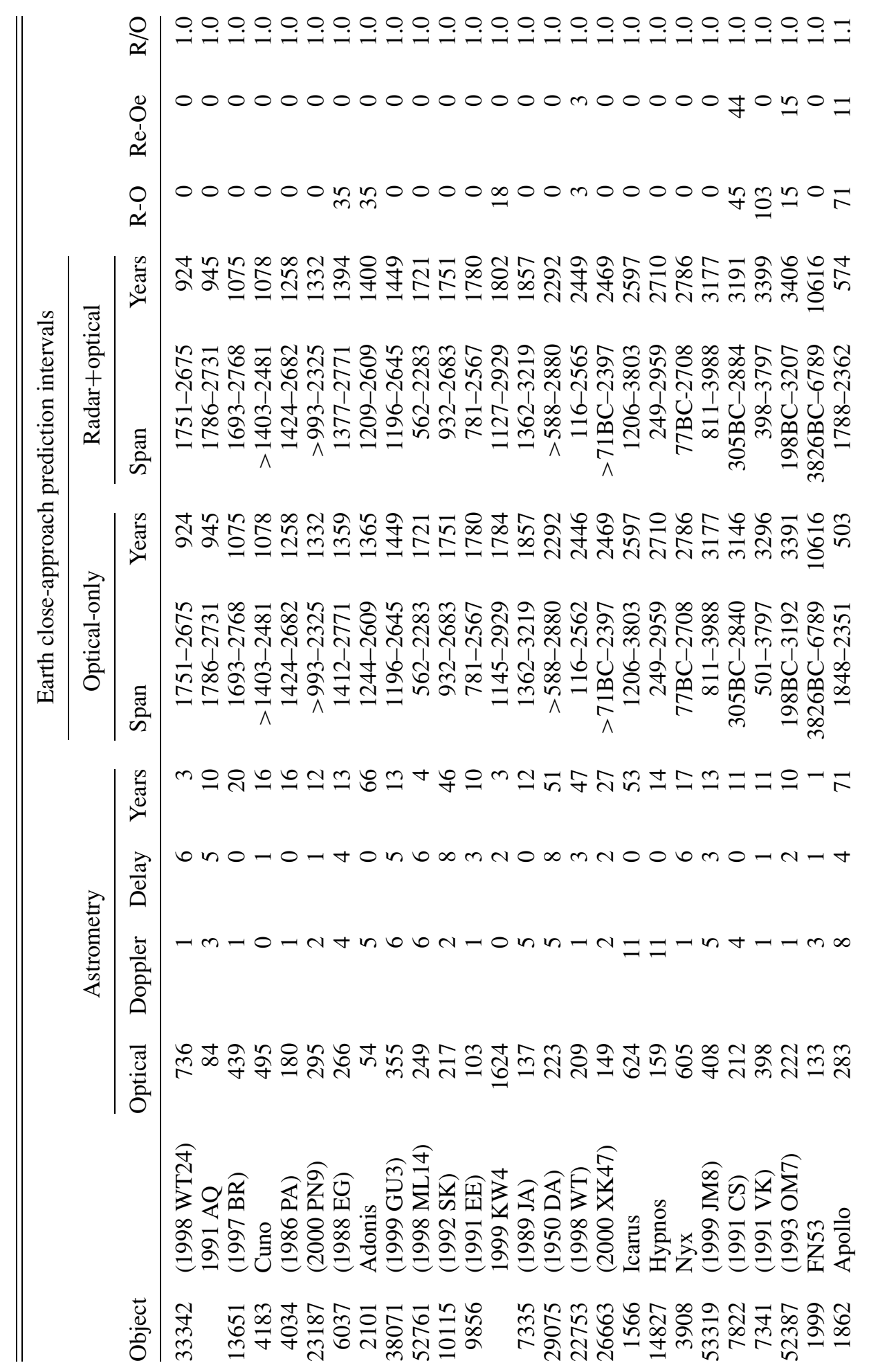




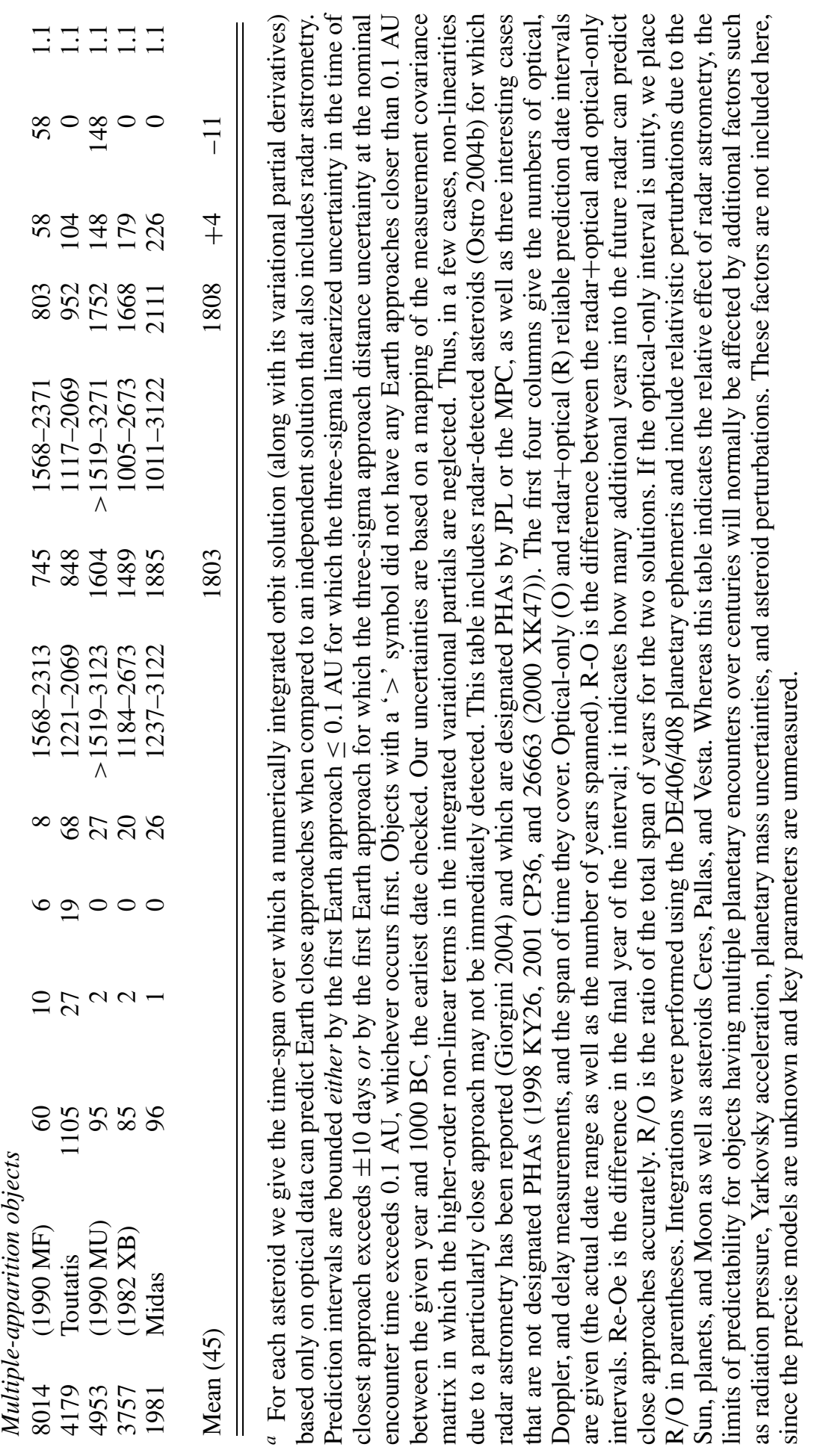


during the discovery apparition, radar astrometry combined with optical data provided an interval of 1183 years. It required the recovery of 1998 ML14 before an optical-only solution yielded a prediction interval comparable to the discovery apparition combined with radar. Similarly, for 1999 FN53, discovery apparition radar indicated an 8257-year window not possible with optical data alone until the object was recovered about half an orbit period later.

For multiple-apparition objects, radar does not significantly extend the interval, which often is terminated centuries from the present era by one or more close planetary approaches whose detailed geometry simply cannot be discerned by any present-day data type. Nevertheless, radar improves the accuracy of multiapparition orbits. A prime example is 1950 DA: the same upper-limit of AD 2880 exists whether or not radar is included in the multiple-apparition solution. However, including radar revealed a non-negligible impact potential in 2880 not apparent in optical solutions. This was because radar astrometry eliminated a bias in the optical data and reduced the 2880 uncertainty region by about $20 \%$ as compared to the optical only solution, resulting in the potential hazard detection (see Section 4.2).

In seven of the 45 multi-apparition cases, radar astrometry actually reduced the interval of prediction, while 17 cases were slightly extended. These disparate effects arise because the different nominal orbits for the optical and optical+radar solutions have slightly different planetary encounter circumstances, so their uncertainty regions increase in different ways. Thus the net effect of radar for these multi-apparition cases is to correct the length of the optically predicted interval, suggesting that if any optical-only orbit were to reveal a potentially hazardous close approach, it would be highly desirable to get radar astrometry to check the prediction.

\section{Radar and collision probability prediction}

For newly discovered NEOs, a collision probability is now routinely estimated (Milani et al. 2002) for close Earth approaches. This probability is combined with the asteroid's estimated diameter and the time until the approach to rate the relative degree of hazard using the Palermo Technical Scale (Chesley et al. 2002). The Jet Propulsion Laboratory's Sentry program maintains a "risk page" (Chesley 2004) which lists objects found to have a potential for impact within the next 100 years. However, for newly discovered objects, the limited number of initial astrometric observations typically does not permit accurate trajectory prediction. When an object's optical astrometric arc is only days or weeks long, the orbit is so uncertain that a potentially hazardous close approach cannot be distinguished from a harmless one or even a non-existent one. The object is placed on the Sentry page, then 
Table 3.5 Simulated impacting orbit

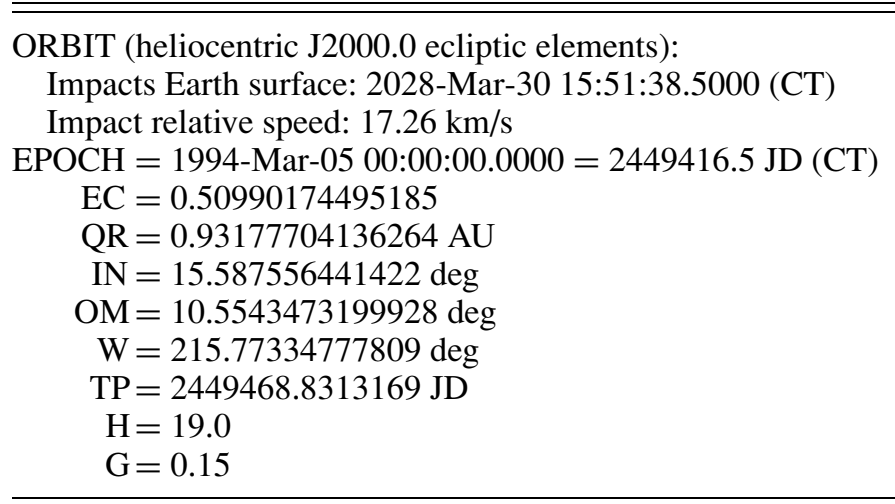

typically removed later, when additional optical astrometry is obtained and the span of observations is extended. However, almost as a rule, objects on the Sentry page have not been observed with radar.

\subsection{A simulated impact scenario}

If an asteroid is on collision course with Earth, this fact will be recognized much sooner with radar data than without it. To examine the possible progression of optical-only and radar+optical impact probability estimates prior to a collision, we constructed a simulation as follows.

First, from the initial set of statistically possible trajectories for a recently discovered asteroid, we selected an orbit that has a 2028 approach to within two Earth radii, a 1994 approach when it could have been discovered, and two postdiscovery periods of visibility. That orbit was adjusted so as to change the 2028 close approach into an impact. We adopted an absolute visual magnitude of $H=19$, which corresponds to an object with a diameter between 420 and $940 \mathrm{~m}$ and having a discovery-apparition peak brightness of magnitude 14 . Thirteen years after discovery, the asteroid brightens to magnitude 19, so recovery would be possible. Subsequent additional observing opportunities exist, but are less favorable since the object does not again get brighter than 20th magnitude until 9 weeks before impact. Radar observations would be possible during the discovery apparition, but then not again until 2 weeks prior to impact. Table 3.5 gives the impacting orbit and Table 3.6 lists observing opportunities.

We then simulated optical astrometry using the impacting reference trajectory and a Gaussian residual noise model in which the residual mean and standard 
Table 3.6 Observing opportunities for the simulation

\begin{tabular}{|c|c|c|c|c|c|}
\hline $\begin{array}{l}\text { Years since } \\
\text { discovery }\end{array}$ & & Date/time & $\begin{array}{l}\text { Visual } \\
\text { brightness } \\
\text { (magnitude) }\end{array}$ & $\begin{array}{r}\text { Radar } \\
\text { SNR }\end{array}$ & Comments \\
\hline \multirow[t]{7}{*}{0} & \multirow{7}{*}{1994} & Mar 10 & 16.7 & - & Optical discovery \\
\hline & & Mar 20 & 15.1 & 532 & Arecibo start \\
\hline & & Mar 27 & 14.0 & 17791 & Last day in Arecibo window \\
\hline & & Mar 28 & 14.3 & 1064 & Goldstone start \\
\hline & & Mar 30 & 15.2 & 455 & Goldstone stop \\
\hline & & Apr 30 & 19.5 & - & $\begin{array}{l}\text { Last optical data (no impact } \\
\text { detection) }\end{array}$ \\
\hline & & Oct 14 & 22.0 & - & $\begin{array}{l}\text { Last optical data (if impact } \\
\text { detection) }\end{array}$ \\
\hline \multirow[t]{3}{*}{13} & \multirow[t]{3}{*}{2007} & Apr 19 & 20.0 & - & Optical recovery \\
\hline & & Jul 17 & 19.0 & - & Peak brightness \\
\hline & & Oct 15 & 22.0 & - & $\begin{array}{l}\text { Last optical data (impact } \\
\text { detection) }\end{array}$ \\
\hline 20 & 2014 & Dec 21 & 21.6 & - & \\
\hline \multirow[t]{2}{*}{21} & \multirow[t]{2}{*}{2015} & Feb 21 & 20.0 & - & \multirow[t]{2}{*}{ Peak brightness } \\
\hline & & Oct 14 & 22.0 & - & \\
\hline \multirow[t]{12}{*}{34} & \multirow[t]{12}{*}{2028} & Jan 22 & 20.0 & - & Optical recovery \\
\hline & & Mar 16 & 16.0 & 15 & Goldstone detection possible \\
\hline & & Mar 30 & 9.5 & & \\
\hline & & $12: 49: 56$ & 6.0 & & Dark-sky naked eye visibility \\
\hline & & $14: 01: 13$ & 5.0 & & \\
\hline & & $14: 45: 55$ & 4.0 & & \\
\hline & & $15: 12: 39$ & 3.0 & & \\
\hline & & $15: 29: 47$ & 2.0 & & \\
\hline & & $15: 40: 14$ & 1.0 & & \\
\hline & & $15: 46: 32$ & 0.0 & & \\
\hline & & $15: 50: 16$ & -1.0 & & \\
\hline & & $15: 51: 38$ & - & & Surface impact \\
\hline
\end{tabular}

deviation for each reporting site's astrometry was based on the actual observing results for 1994 AW7. We simulated radar data for Arecibo and Goldstone using the predicted SNRs to determine observing windows and potential measurement accuracy, adjusting the astrometry to emulate the residual statistics for previous radar campaigns.

Table 3.7 shows the impact probability that would be predicted for each of several cases with different amounts of discovery-apparition radar astrometry. A typical optical campaign at discovery (case B) does not show an unusual impact risk after 50 days of observations. However, if just two radar measurements are made 10 days after discovery (case C), the likelihood of a very close approach immediately becomes evident, along with a non-negligible impact probability. Comparison of 


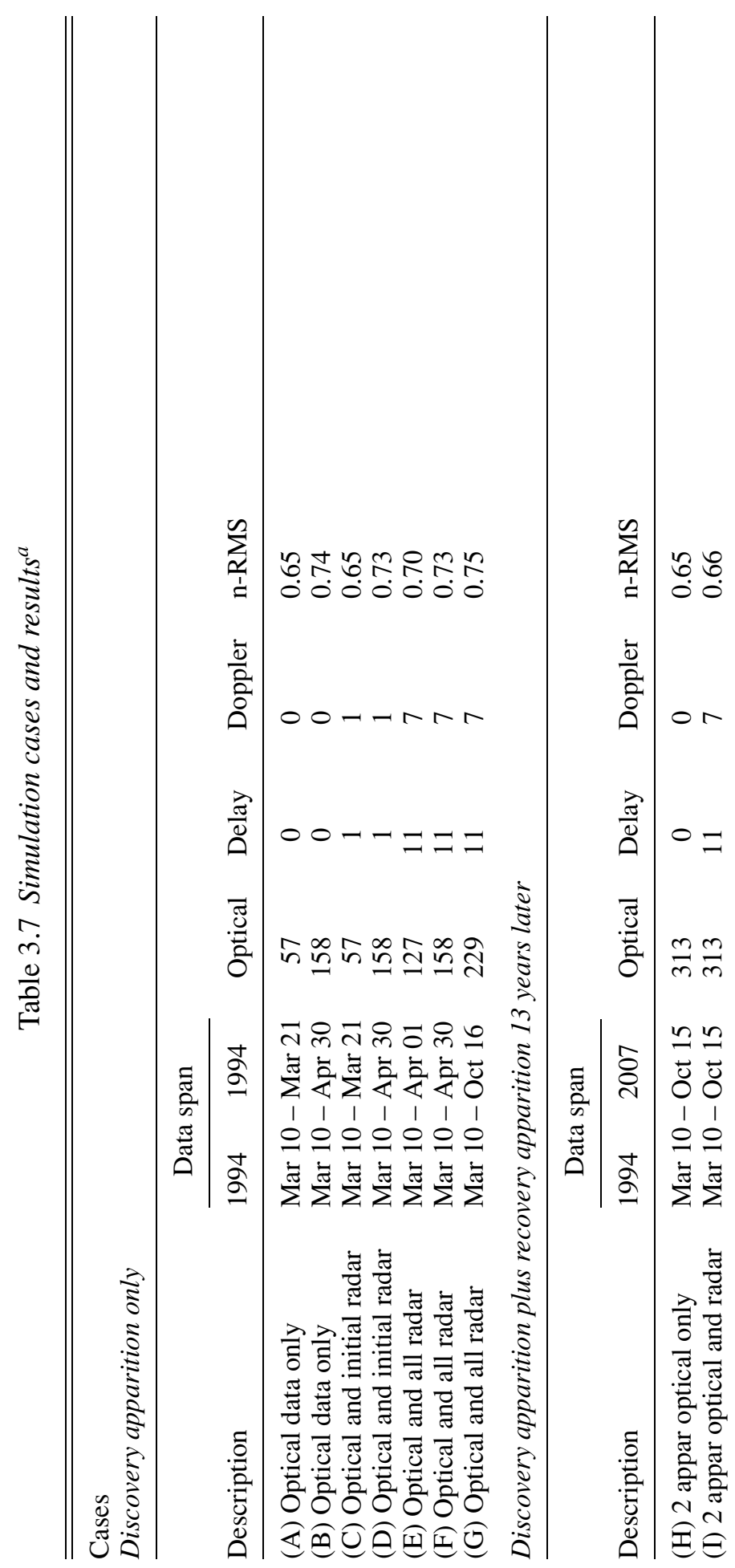




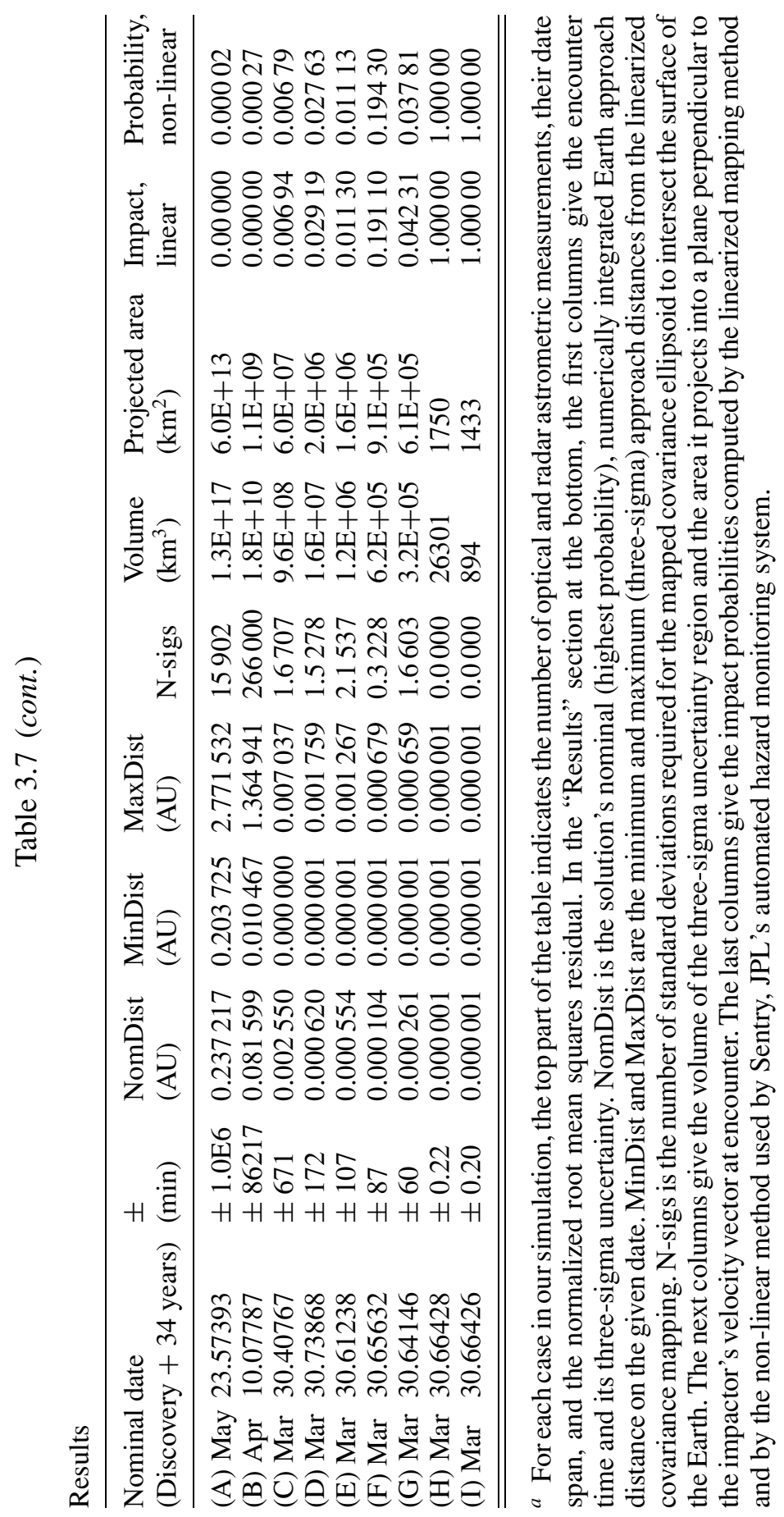


cases $\mathrm{A}$ and $\mathrm{C}$ reveals that after the first two radar measurements, the volume of the uncertainty region is nine orders of magnitude smaller with the radar+optical orbit than with the optical-only orbit.

At the conclusion of case B's 50-day observing window, a $0.027 \%$ impact probability is indicated by the optical-only solution. This is noteworthy, but not unusual for single-apparition objects - there currently are four objects on the Sentry Risk Page with a comparable impact probability. However, with the radar astrometry (case F), a 19\% impact probability is indicated at the same point in time. Radar reduces the volume of the uncertainty region at the encounter by five orders of magnitude compared to the optical-only case B. A $19 \%$ impact probability would attract additional resources and would extend the window of optical observability several months, down to at least magnitude 22 (case G). Due to marginally greater bias and noise in the simulated data as the target fades from view, the additional optical astrometry moves the solution's nominal close approach slightly further away from the Earth, decreasing the impact probability estimate.

If instead there is no radar data at the discovery apparition, recovery would probably still occur during the optically favorable apparition 13 years after discovery. If so, two such apparitions of optical data conclusively identify the impact event whether or not radar data is available (cases $\mathrm{H}$ and I), although the radar data reduces the volume of the uncertainty region by a factor of 29 compared to a solution based only on two apparitions of optical data. However, if the recovery does not occur, the next good opportunity to recover the object and clarify the impact risk, or perhaps to first become aware of it, would be 2 months prior to impact. Radar data during the discovery apparition guarantees the recovery by clearly indicating a high impact risk immediately, providing 34 years of warning instead of 21 years (or possibly only a few weeks).

\subsection{Negative predictions, positive predictions, and warning time}

To a great extent, the dominance of PHA trajectory uncertainties is a temporary one, an artifact of the current discovery phase. Predictions are made for single-apparition objects having a few days or weeks of measurements. The uncertainty region in such cases can encompass a large portion of the inner solar system, thereby generating small but finite impact probabilities that change rapidly as the data arc lengthens, or if high-precision radar delay and Doppler measurements can be made. Impact probabilities in such cases are effectively a statement that the motion of the asteroid is so poorly known that the Earth cannot avoid passing through the asteroid's large uncertainty region - hence the apparent impact "risk." As optical measurements are made, the region shrinks. The resulting change in impact probability, up or down, is effectively a statement about where the asteroid won't be - a "negative 
prediction" - rather than a "positive prediction" of where it will be. This is due to the modest positional precision of optical measurements.

In contrast, radar measurements naturally provide strong constraints on the motion and hence "positive predictions" about where an asteroid will be decades and often centuries into the future. Thus radar measurements substantially open the time-window of positive predictability. However, within a couple of decades, asteroids being found now (but unobserved by radar) will themselves have multiple optical apparitions and similarly be predictable in a positive way over centuries, as radar cases are now. In this way, orbit uncertainties for present-day radar cases illustrate what the situation will be by mid-century for most of the asteroids known today, and presumably for almost all PHAs as large as 1 kilometer.

Unless a significant impact is predicted to occur in the next few decades, mitigation will primarily be an issue for multi-apparition asteroids with decades of observations behind them (since most objects will typically have that much optical astrometry) or for radar-detected objects, with the impact predictions at least centuries in the future. Examination of the next 1000 years will be 30 times as likely to find an actual impactor than examination of the next few decades. This suggests that high-energy mitigation methods may be rendered obsolete before they could be implemented, not by technology, but by the changing nature of orbit predictability as the primary discovery phase ends and observations accumulate. Low-energy methods such as radiation pressure or Yarkovsky modification can potentially be implemented at lower cost, on timescales compatible with the actual hazard.

\section{$4.31950 \mathrm{DA}$}

At this writing, there is only one known NEO with a potentially significant possibility of collision. For 29075 (1950 DA), integrations of the radar-refined orbit by Giorgini et al. (2002) revealed that in 2880 there could be a hazardous approach not indicated in the half-century arc of pre-radar optical data. The current nominal orbit represents a risk as large as $50 \%$ greater than that of the average background hazard due to all other asteroids from now through 2880, as defined by the Palermo Technical Scale (PTS value +0.17). 1950 DA is the only known asteroid whose danger could be above the background level. During the observations, a radar timedelay measurement corrected the optical ephemeris's prediction by $7.9 \mathrm{~km}$, changing an optical-only prediction of a 2880 close approach to a nominal distance of 20 lunar distances into a radar-refined prediction of a nominal distance of 0.9 lunar distances.

The uncertainty in the closeness of 1950 DA's 2880 approach and hence in the probability of a collision (which could be as low as zero or as high as $1 / 300$ ) is due 
Table 3.8 Sources of uncertainty in 1950 DA's position during the 2880 close approach $^{a}$

\begin{tabular}{lc}
\hline \hline Phenomenon & Relative maximum along-track effect \\
\hline Galilean satellites & $1.0(3100 \mathrm{~km}, 4 \mathrm{~min})$ \\
Galactic tide & 2.7 \\
Numerical integration error & 3.2 \\
Solar mass loss & 4.3 \\
Poynting-Robertson drag & 7.7 \\
Solar oblateness & 13.6 \\
61 most perturbing “other” asteroids & 484 \\
Planetary mass uncertainty & 496 \\
Solar radiation pressure & 3613 \\
Yarkovsky effect & 22903 \\
\hline
\end{tabular}

$a$ These factors are normally neglected in asteroid trajectory predictions spanning less than a century. From Giorgini et al. (2002).

to a combination of the factors in Table 3.8. The dominant factor is the Yarkovsky acceleration, which is due to the anisotropic reradiation of absorbed sunlight as thermal energy and depends on the object's mass, size, shape, spin state, and global distribution of optical and thermal properties. Thus, unlike previous cases, predicting a potential 1950 DA impact with the Earth depends mostly on the asteroid's physical characteristics, not initial trajectory measurement. The accelerations are all small, but add up over time and are amplified by 15 close encounters with the Earth or Mars prior to 2880.

The 1950 DA example underscores the fundamental inseparability of the physical properties of NEAs and long-term prediction of their trajectories. The urgency of physically characterizing a threatening object naturally would increase as estimates of the collision probability rise and mitigation is transformed from a hypothetical possibility to an engineering requirement. If we take the hazard seriously, physical characterization of these objects deserves high priority.

\section{Physical characterization}

\subsection{Images and physical models}

With adequate orientational coverage, delay-Doppler images can be used to construct three-dimensional models (e.g., Hudson et al. 2000), to define the rotation state, and to constrain the internal density distribution. Even a single echo spectrum jointly constrains the target's size, rotation period, and sub-radar latitude. A series of Doppler-only echo spectra as a function of rotation phase can constrain the location 
of the center of mass with respect to a pole-on projection of the asteroid's convex envelope (e.g., Benner et al. 1999a). For objects in a non-principal-axis spin state, the hypothesis of uniform internal density can be tested directly (Hudson and Ostro 1995). Given a radar-derived model and the associated constraints on an object's internal density distribution, one can use a shape model to estimate the object's gravity field and hence its dynamical environment, as well as the distribution of gravitational slopes on the surface, which can constrain regolith depth and interior configuration.

For most NEAs, radar is the only Earth-based technique that can make images with useful spatial resolution. Therefore, although a sufficiently long, multiapparition optical astrometric time base might provide about as much advance warning of a possibly dangerous close approach as a radar+optical data set, the only way to compensate for a lack of radar images is with a space mission.

\subsection{Extreme diversity}

As reviewed by Ostro et al. (2002), NEA radar has revealed both stony and metallic objects, principal-axis and complex rotators, very smooth and extraordinarily rough surfaces, objects that must be monolithic and objects that almost certainly are not, spheroids and highly elongated shapes, objects with complex topography and convex objects virtually devoid of topography, contact binaries, and binary systems. Figure 3.2 illustrates some of the diversity of NEAs. Obviously it is useless to talk about the physical characteristics of a "typical" PHA.

\subsection{Surface roughness and bulk density}

Porous, low-strength materials are very effective at absorbing energy (Asphaug et al. 1998). The apparently considerable macroporosity of many asteroids (Britt et al. 2002) has led Holsapple (2002) to claim that impact or explosive deflection methods may be ineffective, even for a non-porous asteroid if it has a low-porosity regolith only a few centimeters deep: "That leaves the low force, long time methods. However, even in those cases the problems of anchoring devices to the surface may make them very difficult."

The severity of surface roughness would be of concern to any reconnaissance mission designed to land or gather samples. The wavelengths used for NEAs at Arecibo $(13 \mathrm{~cm})$ and Goldstone $(3.5 \mathrm{~cm})$, along with the observer's control of the transmitted and received polarizations, make radar experiments sensitive to the surface's bulk density and to its roughness at centimeter to meter (cm-to-m) scales (e.g., Magri et al. 2001). An estimate of the surface bulk density offers a safe lower bound on the subsurface bulk density, and hence a lower bound on the 


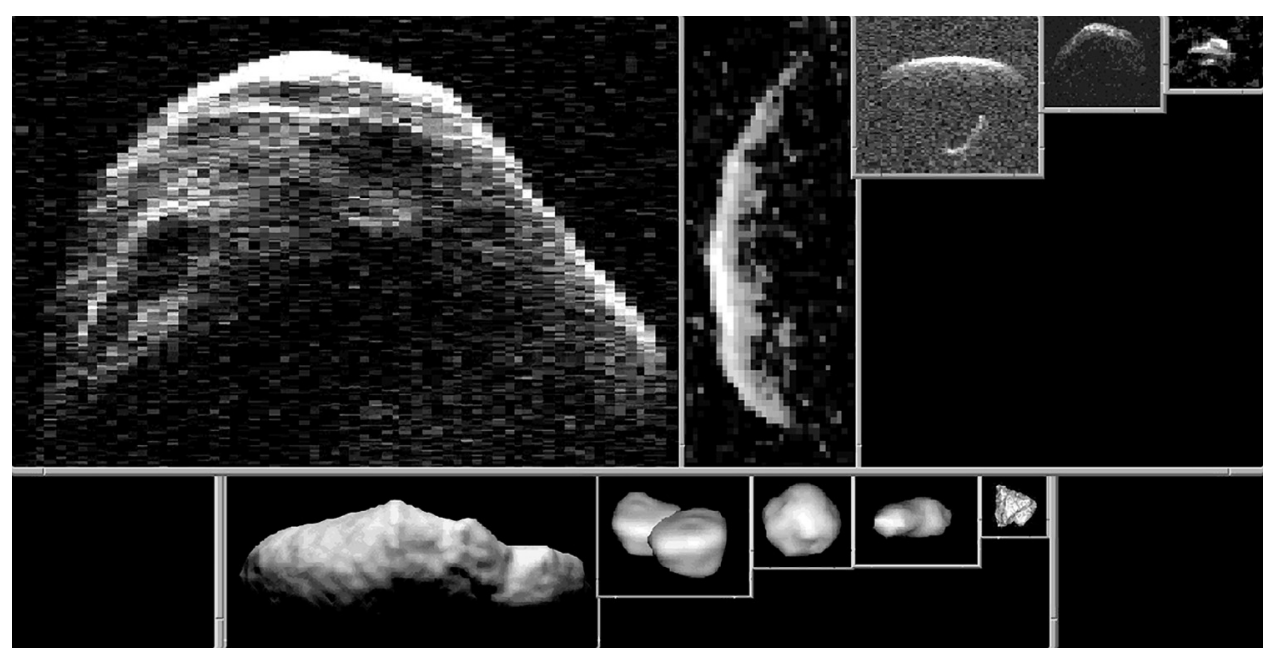

Figure 3.2 Radar delay-Doppler images and shape models. The top collage shows radar images of (left to right) 1999 JM8 (Benner et al. 2002a), Geographos (Ostro et al. 1996), the binary $1999 \mathrm{KW} 4$ (Ostro et al. 2002), 1950 DA (Giorgini et al. 2002), and Golevka (Hudson et al. 2000). The bottom collage shows renderings of shape models of (left to right) Toutatis (Hudson et al. 2003), Castalia (Hudson and Ostro 1994), Nyx (Benner et al. 2002b), Bacchus (Benner et al. 1999b), and Golevka (Hudson et al. 2000). The relative scale of the images and models is approximately correct; Nyx is about $1 \mathrm{~km}$ in diameter.

asteroid's mass. Bulk density is a function of regolith porosity and grain density, so if an asteroid can confidently be associated with a meteorite type, then the average porosity of the surface can be estimated. Values of porosity estimated by Magri et al. (2001) for nine NEAs range from 0.28 to 0.78 , with a mean and standard deviation of $0.53 \pm 0.15$. The current results suggest that most NEAs are covered by at least several centimeters of porous regolith, and therefore the above warning by Holsapple may be valid for virtually any object likely to threaten collision with Earth.

The fact that NEAs' circular polarization ratios (SC/OC) range from near zero to near unity (Fig. 3.3) means that the cm-to-m structure on these objects ranges from negligible to much more complex than any seen by the spacecraft that have landed on Eros (whose SC/OC is about 0.3, near the NEA average), the Moon, Venus, or Mars. 2101 Adonis and 1992 QN (Benner et al. 1997) and 2000 EE104 (Howell et al. 2001) are the extreme examples, with SC/OC near unity.

Ostro et al. (2002) claim that an asteroid's SC/OC can be taken as a crude estimate of the fraction of the surface area covered by roughly wavelength-sized rocks. To what extent might the surface rock coverage be representative of the structural configuration inside the object? NEA surfaces apparently can have rock coverages 


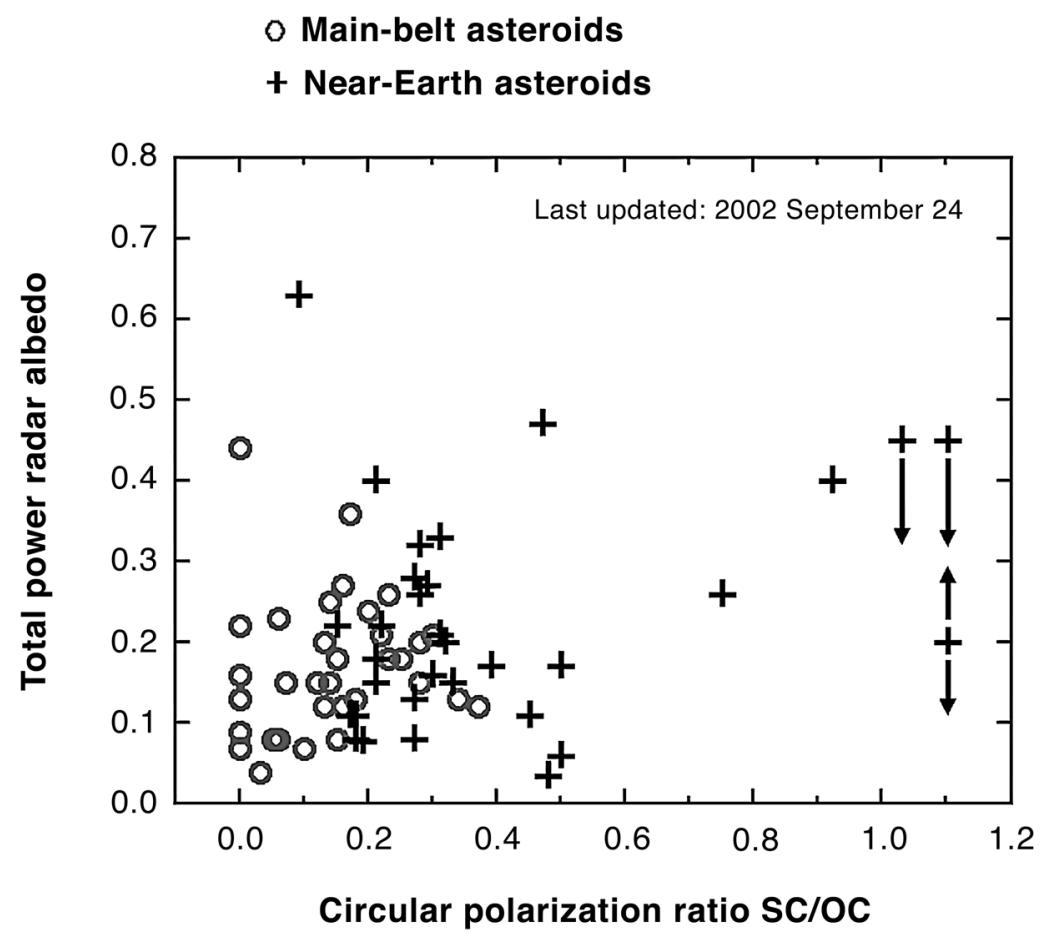

Figure 3.3 Radar properties of NEAs and mainbelt asteroids. The two crosses with single arrows give upper bounds on the albedo; the cross with two arrows indicates an unknown albedo.

anywhere from negligible to total, and NEA interiors apparently can lie anywhere in the Richardson et al. (2002) relative-tensile-strength-vs.-porosity parameter space. Is there any relation between the two? If so, then an object's radar properties may indicate possibilities for its interior and hence for mitigation options. If not, then those properties still constrain options for spacecraft surface operations.

\subsection{Binary NEAs: mass and density}

The most basic physical properties of an asteroid are its mass, its size and shape, its spin state, and whether it is one object or two. The current Arecibo and Goldstone systems are able to identify binary NEAs unambiguously and at this writing have imaged 13 (Margot et al. 2002, and references therein; Nolan et al. 2002; references listed in Ostro 2004b), all of which are designated PHAs. Current detection statistics, including evidence from optical lightcurves (Pravec 2004) suggest that between $10 \%$ and $20 \%$ of PHAs are binary systems. 
Analysis of echoes from these binaries is yielding our first measurements of PHA densities. Delay-Doppler images of 2000 DP107 (Margot et al. 2002) reveal a 800-m primary and a 300-m secondary. The orbital period of $1.767 \mathrm{~d}$ and semimajor axis of $2620 \pm 160 \mathrm{~m}$ yield a bulk density of $1.7 \pm 1.1 \mathrm{~g} \mathrm{~cm}^{-3}$ for the primary. DP107 and the five other radar binaries have spheroidal primaries spinning near the breakup-point for strengthless bodies. Whether binaries' components were mutually captured following a highly dispersive impact into a much larger body (Richardson et al. 2002, and references therein) or formed by tidal disruption of an object passing too close to an inner planet (Margot et al. 2002), it seems likely that the primaries are unconsolidated, gravitationally bound aggregates, so Holsapple's warning applies to them.

\subsection{Radar investigations, mission design, and spacecraft navigation}

Whether a PHA is single or binary, mitigation will involve spacecraft operations close to the object. Maneuvering near a small object is a non-trivial challenge, because of the weakness and complexity of the gravitational environment (Scheeres et al. 2000). Maneuvering close to either component of a binary system would be especially harrowing.

The instability of close orbits looms as such a serious unknown that unless we have detailed information about the object's shape and spin state, it would be virtually impossible to design a mission capable of autonomous navigation close to the object. Control of a spacecraft orbiting close to an asteroid requires knowledge of the asteroid's location, spin state, gravity field, size, shape and mass, as well as knowledge of any satellite bodies that could pose a risk to the spacecraft. Radar can provide information on all these parameters. Knowledge of the target's spin state as well as its shape (and hence nominal gravity harmonics under the assumption of uniform density) (Miller et al. 1999) would permit design of stable orbits immune to escape or unintended surface impact. (Upon its arrival at Eros, the NEAR Shoemaker spacecraft required almost 2 months to refine its estimate of the gravity field enough to ensure reliable close-approach operations.)

If it turns out to be necessary to have a sequence of missions beginning with physical reconnaissance and ending with a deflection, then a radar-derived physical model would speed up this process, reduce its cost, decrease complexity in the design and construction of the spacecraft, and improve the odds of successful mitigation. A reduced need for contingency fuel could be significant enough to allow a smaller launch vehicle for the mission. For example, the result might save $\$ 100$ million via a switch from a Titan III launch vehicle to a Titan IIS, or \$200 million for a switch from a Titan IV to a Titan III. The ability of prior radar 
reconnaissance to reduce mission cost, complexity and risk was embraced by the Department of Defense in their proposed Clementine II multiple-flyby mission (Hope et al. 1997), all of whose candidate targets either had already been observed with radar (Toutatis, Golevka) or were radar observable prior to encounter (1987 OA, 1989 UR).

Ironically, although PHAs include the lowest- $\Delta V$ rendezvous targets in the solar system, Japan's Hayabusa (MUSES-C) sample-return mission to 25143 Itokawa (1998 SF36) is the world's first rendezvous mission to a PHA. Results of radar imaging of that asteroid (Ostro et al. 2001) are being used by the Japanese Institute of Space and Astronautical Science in planning for the late 2005 rendezvous, and radar observations during the asteroid's mid-2004 close approach will be used for navigational assistance and to refine the model derived from the 2001 images. Radar-derived shape models of small NEAs have made it possible to explore the evolution and stability of close orbits (e.g., Scheeres et al. 1996, 1998), and this experience is currently being applied to Hayabusa.

Radar refinement of physical properties and radar refinement of orbits are very tightly coupled: shape modeling necessarily involves refinement of the delayDoppler trajectory of the center of mass through the observing ephemerides. With very precise radar astrometry, a spacecraft lacking onboard optical navigation could be guided into orbit around, or collision course with, an asteroid. For example, consider how Goldstone observations shrunk the positional error ellipsoid of Geographos, an object already heavily observed by optical telescopes, just prior to a planned Clementine flyby of that target on August 31, 1994 (Ostro 1996). Before Goldstone ranging observations carried out during August 2829 , the overall dimension of the positional error ellipsoid was $\sim 11 \mathrm{~km}$. The radar astrometry collapsed the ellipsoid's size along the line of sight to several hundred meters, so its projection toward Clementine on its inbound leg would have been $11 \times 2 \mathrm{~km}$. Goldstone-VLA radar aperture synthesis angular astrometry (see discussions by de Pater et al. 1994, and Hudson et al. 2000), could have shrunk the error ellipsoid's longest dimension to about $1 \mathrm{~km}$, about half of Geographos' shortest overall dimension. For less well-observed objects, the gains could be substantially more, as with 1862 Apollo's 3750 km radar range correction.

\subsection{Modeling the efficiency of explosive deflection}

Mitigation scenarios include the use of explosives to deflect the projectile (Ahrens and Harris 1992). However, as demonstrated by Asphaug et al. (1998), the outcome 
of explosive energy transfer to an asteroid or comet (via a bomb or a hypervelocity impact) is extremely sensitive to the pre-existing configuration of fractures and voids, and also to impact velocity. Just as porosity damps shock propagation, sheltering distant regions from impact effects while enhancing energy deposition at the impact point, parts of multi-component asteroids are preserved, because shock waves cannot bridge inter-lobe discontinuities. A radar-derived shape model would allow more realistic investigation (Asphaug et al. 1998) of the potential effectiveness of nuclear explosions in deflecting or destroying a hazardous asteroid.

\subsection{Comets}

The risk of a civilization-ending impact during this century is about the same as the risk of a civilization-ending impact by a long-period comet (LPC) during this millennium. At present, the maximum possible warning time for an LPC impact probably is between a few months and a few years. Comet trajectory prediction is hampered by optical obscuration of the nucleus and by uncertainties due to timevarying, non-gravitational forces. Comets are likely to be very porous aggregates, so concern about the ineffectiveness of explosive deflection is underscored in the case of comets.

Radar reconnaissance of an incoming comet would be the most reliable way to estimate the size of the nucleus (Harmon et al. 1999), could reveal the prevalence of centimeter-and-larger particles in the coma (Harmon et al. 1989, 1997), and would be valuable for determining the likelihood of a collision.

\section{Recommendations}

How much effort should be made to make radar observations of NEAs? For newly discovered objects, it is desirable to guarantee recovery and to ensure accurate prediction of close approaches well into the future, and at least throughout this century. Moreover, a target's discovery apparition often provides the most favorable radar opportunity for decades and hence a unique chance for physical characterization that otherwise would require a space mission. Similarly, even for NEAs that have already been detected, any opportunity offering a significant increment in echo strength and hence imaging resolution should be exploited. Binaries and non-principal-axis rotators, for which determination of dynamical and geophysical properties requires a long, preferably multi-apparition time base, should be observed extensively during any radar opportunity.

Construction of the proposed Large Synoptic Survey Telescope (LSST) has been endorsed (Belton et al. 2002), in part as a means to extend the Spaceguard Survey's 
90\% completeness goal for kilometer-sized objects down to 300-m objects. However, both Arecibo and Goldstone are already heavily oversubscribed, with only several percent of their time available for asteroid radar. Over the coming decades, it may become increasingly clear that most of the NEO radar reconnaissance that is technically achievable with Arecibo and Goldstone is precluded by the limited accessibility of those instruments, and that a dedicated NEO radar instrument is desirable.

An ideal NEO radar system (Ostro 1997) might consist of two antennas like the 100-m NRAO Greenbank Telescope (GBT, in West Virginia), one with a megawatt transmitter and one just for receiving, separated by a few tens of kilometers, operating at a wavelength of $0.9 \mathrm{~cm}$ (Ka band). Each antenna's gain could be $88 \mathrm{~dB}$, compared to $73.5 \mathrm{~dB}$ for Arecibo. A two-antenna (bistatic) configuration would eliminate the frequent transmit/receive alternation and klystron power cycling required in single-antenna observations of NEOs and would double the available integration time. The antennas would be fully steerable, so any object could be tracked at least several times longer than at Arecibo. The combination of all these factors would make this dedicated NEO radar an order of magnitude more sensitive than the upgraded Arecibo telescope. The capital cost of building this system now, as calibrated by the GBT experience, would be within $10 \%$ of $\$ 180$ million, comparable to the cost of a small Discovery mission and very close to the estimated cost of the LSST.

\section{Acknowledgment}

This research was conducted at the Jet Propulsion Laboratory, California Institute of Technology, under contract with the National Aeronautics and Space Administration (NASA). S. Ostro was partially supported by the Steven and Michele Kirsch Foundation.

\section{References}

Ahrens, T. J. and Harris, A. W. 1992. Deflection and fragmentation of near-Earth asteroids. Nature 360, 429-433.

Asphaug, E., Ostro, S. J., Hudson, R. S., et al. 1998. Disruption of kilometre-sized asteroids by energetic collisions. Nature 393, 437-440.

Beatty, J. K. 2002. Arecibo radar gets 11th-hour reprieve. Sky Publishing Corp. [cited April 23, 2004]. Available online at http://skyandtelescope.com/news/archive/article_285_1.asp.

Belton, M. J., Porco, C., A'Hearn, M., et al. (2002). New Frontiers in the Solar System: An Integrated Exploration Strategy Solar System Exploration Survey. Washington, DC: National Research Council. 
Benner, L. A. M., Ostro, S. J., Giorgini, J. D., et al. 1997. Radar detection of near-Earth asteroids 2062 Aten, 2101 Adonis, 3103 Eger, 4544 Xanthus, and 1992 QN. Icarus 130, 296-312.

Benner, L. A. M., Ostro, S. J., Rosema, K. D., et al. 1999a. Radar observations of asteroid 7822 (1991 CS). Icarus 137, 247-259.

Benner, L. A. M., Hudson, R. S., Ostro, S. J., et al. 1999b. Radar observations of asteroid 2063 Bacchus. Icarus 139, 309-327.

Benner, L. A. M., Ostro, S. J., Nolan, M. C., et al. 2002a. Radar observations of asteroid 1999 JM8. Meteor. and Planet. Sci. 37, 779-792.

Benner, L. A. M., Ostro, S. J., Hudson, R. S., et al. 2002b. Radar observations of asteroid 3908 Nyx. Icarus 158, 379-388.

Britt, D. T., Yeomans, D., Housen, K., et al. 2002. Asteroid density, porosity, and structure. In Asteroids III, eds. W. F. Bottke, A. Cellino, P. Paolicchi, and R. P. Binzel, pp. 485-500. Tucson, AZ: University of Arizona Press.

Chesley, S. R. 2004. Current impact risks. California Institute of Technology, Pasadena [cited April 23, 2004]. Available online at http://neo.jpl.nasa.gov/risks.

Chesley, S. R., Chodas, P. W., Milani, A., 2002. Quantifying the risk posed by potential Earth impacts. Icarus 159, 423-432.

de Pater, I., Palmer, P., Mitchell, D. L., et al. 1994. Radar aperture synthesis observations of asteroids. Icarus 111, 489-502.

Giorgini, J. D. 2004. Small-body astrometric radar observations. California Institute of Technology, Pasadena [cited April 23, 2004]. Available online at http://ssd.jpl.nasa.gov/radar_data.html.

Giorgini, J. D., Ostro, S. J., Benner, L. A. M., et. al. 2002. Asteroid 1950 DA's encounter with Earth in 2880: physical limits of collision probability prediction. Science 296, 132-136.

Goldstein, R. M. 1968. Radar observations of Icarus. Science 162, 903-904.

Harmon, J. K., Campbell, D. B., Hine, A. A., et al. 1989. Radar observations of comet IRAS-Araki-Alcock 1983d. Astroph. J. 338, 1071-1093.

Harmon, J. K., Ostro, S. J., Benner, L. A. M., et al. 1997. Comet Hyakutake (C/1996 B2): radar detection of nucleus and coma. Science 278, 1921-1924.

Harmon, J. K., Campbell, D. B., Ostro, S. J., et al. 1999. Radar observations of comets. Planet. Space Sci. 47, 1409-1422.

Holsapple, K. A. (2002). The deflection of menacing rubble pile asteroids. In Extended Abstracts from the NASA Workshop on Scientific Requirements for Mitigation of Hazardous Comets and Asteroids, Arlington, VA, September 3-6, 2002, eds. E. Asphaug and N. Samarasinha. pp. 49-52.

Hope, A. S., Kaufman, B., Dasenbrock, R., et al. 1997. A Clementine II mission to the asteroids. In Dynamics and Astrometry of Natural and Artificial Celestial Bodies, Proceedings of IAU Colloquium 165, eds. I. M. Wytrzyszczak, J. H. Lieske, and R. A. Feldman, pp. 183-190. Dordrecht, The Netherlands: Kluwer.

Howell, E. S., Nolan, M. C., DeRemer, L., et al. 2001. Arecibo radar observations of near-Earth asteroid 2000 EE104. Bull. Am. Astron. Soc., 33, 1153.

Hudson, R. S. and Ostro, S. J. 1994. Shape of asteroid 4769 Castalia (1989 PB) from inversion of radar images. Science 263, 940-943.

1995. Shape and non-principal-axis spin state of asteroid 4179 Toutatis. Science 270, 84-86.

Hudson, R. S., Ostro, S. J., Jurgens, R. F., et al. 2000. Radar observations and physical modeling of asteroid 6489 Golevka. Icarus 148, 37-51. 
Hudson, R. S., Ostro, S. J., and Scheeres, D. J. 2003. High-resolution model of asteroid 4179 Toutatis. Icarus 161, 348-357.

Magri, C., Consolmagno, G. J., Ostro, S. J., et al. 2001. Radar constraints on asteroid regolith compositions using 433 Eros as ground truth. Meteor. and Planet. Sci. 36, 1697-1709.

Margot, J. L., Nolan, M. C., Benner, L. A. M., et al. 2002. Binary asteroids in the near-Earth object population. Science 296, 1445-1448.

Milani, A., Chesley, S. R., Chodas, P. W., et al. 2002. Asteroid close approaches: analysis and potential impact detection. In Asteroids III, eds. W. F. Bottke, A. Cellino, P. Paolicchi, and R. P. Binzel, pp. 55-69. Tucson, AZ: University of Arizona Press.

Miller, J. K., Antreasian., P. J., Gaskell, R. W., et al. 1999. Determination of Eros physical parameters for near-Earth asteroid rendezvous orbit phase navigation. Am. Astron. Soc. Paper no. 99-463.

Morrison, D., Harris, A. W., Sommer, G., et al. 2002. Dealing with the impact hazard. In Asteroids III, eds. W. F. Bottke, A. Cellino, P. Paolicchi, and R. P. Binzel, pp. 739-754. Tucson, AZ: University of Arizona Press.

Nolan, M. C., Howell, E. S., Ostro, S. J., et al. 2002. 2002 KK_8. IAU Circ. No. 7921.

Ostro, S. J. 1994. The role of groundbased radar in near-Earth object hazard identification and mitigation. In Hazards due to Comets and Asteroids, eds. T. Gehrels, pp. 259-282. Tucson, AZ: University of Arizona Press.

1996. Radar astrometry of asteroids, comets and planetary satellites. In Dynamics and Ephemerides of the Solar System, Proceedings of IAU Symposium 172, eds. S. Ferraz-Mello, B. Morando, and J.-E. Arlot, pp. 365-372. Dordrecht, The Netherlands: Kluwer.

1997. Radar reconnaissance of near-Earth objects at the dawn of the next millennium. Ann. New York Acad. Sci. 822, 118-139.

2004a. Echo strength predictions. California Institute of Technology, Pasadena [cited April 23, 2004]. Available online at http://echo.jpl.nasa.gov/ ostro/snr/.

2004b. Radar-detected asteroids. California Institute of Technology, Pasadena [cited April 23, 2004]. Available online at http://echo.jpl.nasa.gov/ asteroids/index.html.

Ostro, S. J., Campbell, D. B., Chandler, J. F., et al. 1991. Asteroid radar astrometry. Astron. J. 102, 1490-1502.

Ostro, S. J., Jurgens, R. F., Rosema, K. D., et al. 1996. Radar observations of asteroid 1620 Geographos. Icarus 121, 44-66.

Ostro, S. J., Benner, L. A. M., Nolan, M. C., et al. 2001. Radar observations of asteroid 25143 (1998 SF36). Bull. Am. Astron. Soc. 33, 1117.

Ostro, S. J., Hudson, R. S., Benner, L. A. M., et al. 2002. Asteroid radar astronomy. In Asteroids III, eds. W. F. Bottke, A. Cellino, P. Paolicchi, and R. P. Binzel, pp. 151-168. Tucson, AZ: University of Arizona Press.

Pettengill, G. H., Shapiro, I. I., Ash, M. E., et al. 1969. Radar observations of Icarus Icarus, 10, 432-435.

Pravec, P. 2004. Binary near-Earth asteroids. Ondrejov Observatory [cited April 23, 2004]. Available online at http://www.asu.cas.cz/ asteroid/binneas.htm.

Richardson, D. C., Leinhardt, Z. M., Melosh, H. J., et al. 2002. Gravitational aggregates: evidence and evolution. In Asteroids III, eds. W. F. Bottke, A. Cellino, P. Paolicchi, and R. P. Binzel, pp. 501-515. Tucson, AZ: University of Arizona Press.

Scheeres, D. J., Ostro, S. J., Hudson, R. S., et al. 1996. Orbits close to asteroid 4769 Castalia. Icarus 121, 67-87.

1998. Dynamics of orbits close to asteroid 4179 Toutatis. Icarus 132, 53-79. 
Scheeres, D. J., Williams, B. G., and Miller, J. K. 2000. Evaluation of the dynamic environment of an asteroid: applications to 433 Eros. J. Guidance, Control and Dynamics 23, 466-475.

Shapiro, I. I., Ash, M. E., and Smith, W. B. 1968. Icarus: further confirmation of the relativistic perihelion precession. Phys. Rev. Lett. 20, 1517-1518.

Yeomans, D. K., Ostro, S. J., and Chodas, P. W. 1987. Radar astrometry of near-Earth asteroids. Astron. J. 94, 189-200.

Zellner, B. 1979. Asteroid taxonomy and the distribution of the compositional types. In Asteroids, ed. T. Gehrels, pp. 783-806. Tucson, AZ: University of Arizona Press. 